\title{
Is upper limb virtual reality training more intensive than conventional training for patients in the subacute phase after stroke? An analysis of treatment intensity and content
}

Iris Brunner ${ }^{1,2^{*}}$ (D) Jan Sture Skouen ${ }^{1,2}$, Håkon Hofstad ${ }^{2}$, Jörg Aßmuss ${ }^{3}$, Frank Becker ${ }^{4,5}$, Hanne Pallesen ${ }^{6}$, Liselot Thijs ${ }^{7}$ and Geert Verheyden ${ }^{8}$

\begin{abstract}
Background: Virtual reality (VR) training is thought to improve upper limb (UL) motor function after stroke when utilizing intensive training with many repetitions. The purpose of this study was to compare intensity and content of a VR training intervention to a conventional task-oriented intervention (CT).

Methods: A random sample of 50 video recordings was analyzed of patients with a broad range of UL motor impairments (mean age 61y, 22 women). Patients took part in the VIRTUES trial and were randomized to either VR or $\mathrm{CT}$ and stratified according to severity of paresis. A standardized scoring form was used to analyze intensity, i.e. active use of the affected UL expressed in \% of total time, total active time and total duration of a training session in minutes, content of training and feedback. Two raters collected data independently. Linear regression models as well as descriptive and graphical methods were used.

Results: Patients in the VR group spent significantly more time actively practicing with an activity rate of 77.6 (8.9) \% than patients in the CT 67.3 (13.9) \%, ( $p=.003)$. This difference was attributed to the subgroup of patients with initially severe paresis $(n=22)$. While in VR severely impaired patients spent $80.7 \%$ (4.4\%) of the session time actively; they reached 60.6 (12.1) \% in CT. VR and CT also differed in terms of tasks and feedback provided.
\end{abstract}

Conclusion: Our results indicate that patients with severely impaired UL motor function spent more time actively in VR training, which may influence recovery. The upcoming results of the VIRTUES trial will show whether this is correlated with an increased effect of VR compared to CT.

Trial registration: ClinicalTrials.gov NCT02079103, February 27, 2014.

Keywords: Virtual reality, Stroke, Upper limb, Neurorehabilitation, Motor function, Physical therapy, Occupational therapy

\footnotetext{
* Correspondence: Iris.Brunner@uib.no

'Department of Global Public Health and Primary Care, University of Bergen,

Kalfarveien 31, 5018 Bergen, Norway

${ }^{2}$ Department of Physical Medicine and Rehabilitation, Haukeland University

Hospital, Bergen, Norway

Full list of author information is available at the end of the article
} 


\section{Background}

Intensity and repetition have been identified as key factors for promoting neural plasticity [1]. It has been stated that Virtual Reality (VR) training using either specially developed systems or off-the-shelf gaming consoles provides the opportunity to achieve many repetitions, salient stimuli and engages the patients in a motivating and intense way [2]. Furthermore, VR is supposed to deliver taskspecific training and multi-sensory stimulation [3]. Some evidence has been found that supports the use of VR for improving arm function after stroke [4], although a recent multicenter trial could not corroborate the superiority of a commercial VR gaming system [5]. As VR is becoming progressively more used in neurorehabilitation a more detailed analysis of VR intensity and treatment components is indicated.

Intensity can be expressed as dosage. There is consent that a higher dosage of movement practice can contribute to better outcomes $[6,7]$. However, how the term "dosage" should be defined or which factors of dosage are relevant for improved outcome, is unclear. When examining doseresponse relationships, Lohse et al. [8] found a positive and significant relationship between amounts of therapy provided and motor function improvement after stroke. However, the authors pointed out the need of a more precise measure of active time and repetitions. In a recent review Lang et al. [9] emphasized the need for a deeper understanding of dose-response relationships. Measuring amount of practice in terms of therapy sessions - scheduled or actually conducted - has been widely used, but does not reflect intensity or active time. It has been demonstrated that patients spend less than two-thirds of their treatment sessions actively and that physiotherapists tend to overestimate the amount of active practice [10, 11]. Many VR systems provide a substantial advantage by the integrated registration of time spent actively practicing and other information on training performance $[3,12]$.

Like duration of therapy time, also many repetitions of meaningful and challenging exercises are regarded as beneficial for regaining motor skills after stroke [13]. Timmermans et al. [14] identified 15 components to characterize task-oriented training and examined their relation to effect sizes. They found random and distributed practice, clear functional goals and feedback to be associated with larger effect sizes. These components, however, can be found in both VR and conventional training.

Rand et al. [15] used accelerometers to compare the amounts of purposeful movements elicited in a group of patients with stroke using video games and a control group receiving traditional therapy. They found that playing video games resulted in more purposeful repetitions (median 271) than traditional training (median 48). Also cognitive and emotional involvement, considered as key factors for regaining motor skills, may be facilitated by many VR applications due to their playful character [16-18].

The objective of this study was to compare the intensity, here defined as time spent actively using the affected upper limb, and the content of a VR training intervention and a conventional task-oriented intervention. We hypothesized that the intensity of training was higher in the VR group and that patients in VR would achieve more repetitions.

\section{Methods \\ Design}

Video recordings of 50 patients with impaired upper limb motor function after stroke in five different rehabilitation sites were obtained, 25 of virtual reality (VR) and 25 of conventional arm training (CT). The patients were a consecutive subsample of those who took part in the VIRTUES trial, where they were randomized to either VR training or conventional training for the upper extremity for four weeks, with details described elsewhere [19]. Patients could be included in the main study within three months post stroke if they had a first ever stroke, or former stroke without motor residuals and did not suffer from major cognitive problems (score of $>20$ on MMSE). Inclusion criteria for arm motor function were a score of less than 52 on the Action Research Arm Test (ARAT) and at least 20 degrees of active shoulder extension and abduction. Severe paresis was defined as less than 20 degrees of active wrist and ten degrees of active finger extension, otherwise patients were classified as mild-moderate. Patients were assessed with the Action Research Arm Test (ARAT), Box and Blocks Test (BB) and Functional Independence Measure (FIM) when entering the study. Research staff was asked to videotape one random treatment session during the fourweek treatment period with patients from both VR and conventional training and both severity strata.

\section{Intervention}

The intervention lasted for four weeks with four to five training sessions/week of $45-60$ min duration. Length of an individual session was determined by the patients' general condition and motivation. Patients in both groups were seated at a table during the training session and received individually tailored exercises for arm and hand movements according to their needs and abilities, based on a pre-defined and standardized treatment program.

Patients randomized to the VR group participated in VRbased training with the YouGrabber system (YouRehab Ltd., Switzerland) which comprises wearable data gloves with sensors and training software with different rehabilitation games. The YouGrabber provides a graded training program of task-related exercises that can be performed 
unilaterally or bilaterally. Conventional arm training comprised task-related practice for gross movements and dexterity including different grips and selective finger movements, strength training, stretching, and training in daily life activities. Patients in both groups were encouraged to active training. The speed and the number of repetitions were adapted to patients' actual abilities either automatically by the VR system or the therapist in VR, or the therapist alone in CT.

\section{Video analysis}

The video recordings were analyzed with regard to active training time, total training time, content of training, number of repetitions, and activity rate, defined as the percentage of time spent actively practicing during a therapy session. Time was registered with a stop watch in the conventional training group. Time was stopped when a task was obviously terminated or when no visible activity was present for more than $2 \mathrm{~s}$. In the VR group active time was automatically generated by the system. A scoring form for video analysis was developed including categories used by Timmermans et al. to describe taskoriented training [14]. Other categories were added, such as mobilization, strength training and unimanual/ bimanual training (see Additional file 1). The number of tasks within a training session was registered.

Inspired by procedures applied in former studies by De Wit et al., the following steps were undertaken to assure inter-rater reliability and content validity [20]. Two different raters tried out the scoring form when watching training sessions. They scored independently, compared and discussed their results after each task. As a result, several adjustments of the scoring form were made. During an initial calibration meeting both raters watched training sessions together and compared their assessments immediately. They agreed on procedures to minimize systematic errors. A difference of less than $10 \%$ for the measurement of total active time in one session between the raters was regarded as acceptable. After the initial calibration process the raters watched and scored the video recordings independently. The interrater reliability was examined with ICC statistics with excellent agreement $\left(\mathrm{ICC}_{\text {agreement }}=.98\right)$.

\section{Data analysis}

Descriptive statistics and frequencies were used to describe patient characteristics. To understand the association between treatment and total time (the duration of a training session), active training time (time spent actively during a training session) and activity rate (percentage of time spent actively) we used linear regression models as well as descriptive and graphical methods. We fitted an unadjusted linear regression model with the predictors VR and severe paresis one at a time as well as an adjusted model including both predictors and their interaction. T-tests and Chi-square tests were applied to assess differences in patient characteristics and repetitions. For data analysis SPSS 22 and Matlab 7.10 were used. The significance level was set to 0.05 .

\section{Results}

Video recordings of 50 patients $(n=25$ in VR, $n=25$ in $\mathrm{CT}$ ) were analyzed with regard to total training time, active training time, activity rate and content of training. Patient characteristics are presented in Table 1.

The groupwise mean time measures are presented in Fig. 1 while the results of the comparison of the treatment groups are presented in Table 2 . We observed a significant difference in activity rate of $10.3 \%$ and active time of $6.1 \mathrm{~min}$, while there was no significant difference in total time. This is visible in the univariate analysis, which only shows the differences between VR and CT in the blue graphs in Fig. 1. The multivariate analysis, taking into account the interaction of severity of paresis and kind of intervention received, showed that patients with severe paresis (red) achieved a significantly higher activity rate, while patients with mild paresis (green) did not. The ARAT at study admission had been 7.5 (6.6) for severe and 39.5 (9.6) for patients with mild to moderate paresis. Also active training time and total training time was different for the severely impaired patients only. This effect was significant as shown by the significant interaction in the multivariate analysis.

The content of the training differed in several respects. While the tasks in the VR comprised solely task-related functional movements, tasks in the CT covered a broader range including strength exercises and mobilization. There was no difference between VR and CT with regard to repetitions $(p=0.80)$. However, only discrete functional movements could be counted and not continuous movements which were frequently performed in both groups.

Table 1 Characteristics of patients in each group

\begin{tabular}{llll}
\hline & VR $(n=25)$ & CT $(n=25)$ & $\mathrm{p}$ \\
& Mean (SD) & Mean (SD) & \\
\hline Age, years & $59.6(15.6)$ & $61.6(12.6)$ & .62 \\
Days post stroke & $50.9(19.0)$ & $48.6(24.0)$ & .71 \\
Days after baseline assessment & $22.2(7.3)$ & $21.0(9.7)$ & .77 \\
ARAT (score out of 57) & $28.8(16.1)$ & $23.2(19.0)$ & .29 \\
Box and Blocks (blocks per min.) & $16.6(14.4)$ & $12.4(14.0)$ & .31 \\
FIM (score out of 126) & $91.0(21.3)$ & $97.7(21.5)$ & .28 \\
& $\mathrm{n}$ & $\mathrm{n}$ & \\
Sex (male/female) & $16 / 9$ & $12 / 13$ & .73 \\
Affected arm (left/right) & $15 / 10$ & $12 / 13$ & .57 \\
Paresis (Moderate to mild/severe) & $15 / 10$ & $12 / 13$ & .57 \\
\hline
\end{tabular}

Abbreviations: ARAT Action Research Arm Test, FIM Functional Independence Measure, $V R$ Virtual reality training 


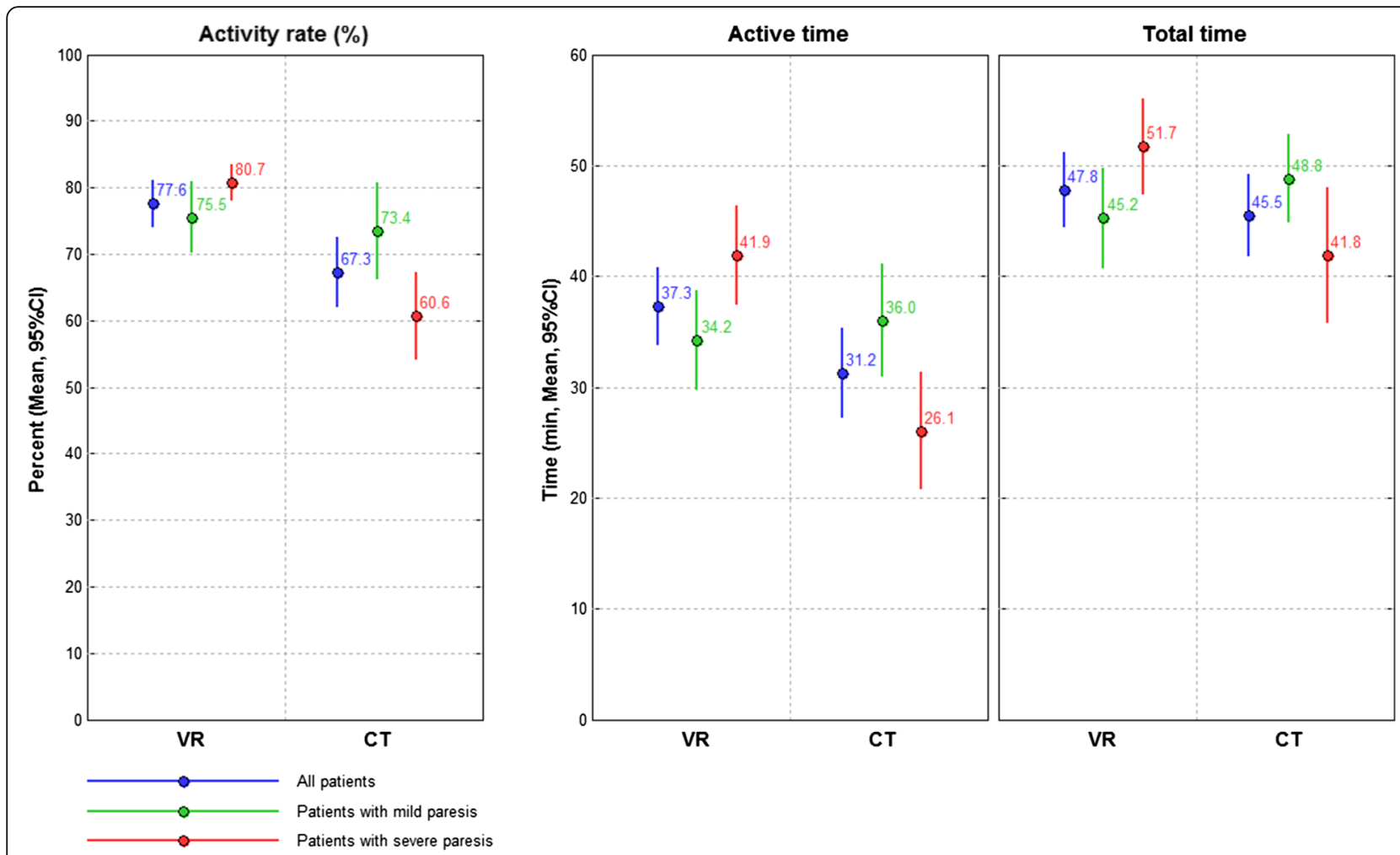

Fig. 1 Activity rate, active time and total time for Virtual Training (VR) and conventional training (CT) for all patients (blue), and subdivided into patients with mild to moderate (green) and severe (red) paresis

Table 3 provides an overview of the content of training in VR and CT. Tasks in VR were shorter, 2-3 min, but frequently repeated during one session and then counted as one task.

\section{Discussion}

VR training resulted in a higher activity rate per training session. This was especially pronounced for patients with severe paresis who also had longer training sessions and more active time in VR than in CT. For patients with mild to moderate paresis, the difference was present, but less pronounced and not statistically significant. Our results suggest that a higher activity rate was easier to reach in the VR group. This is in accordance with what generally is regarded as a main benefit of VR training [2]. The entertaining and persistent character of the training may facilitate a higher training intensity making it easier to achieve a higher activity rate. VR training normally provides a wide range of stimulating tasks. Interestingly, this seems to be particularly relevant for patients with little motor function. Purposeful, taskrelated exercises are regarded as most beneficial for arm motor function after stroke [6]. However, it can be very challenging for therapists to find appropriate active exercises for this patient group and to continue training when the patient shows signs of strain. In suitable VR environments patients experience that even small movements can be translated into purposeful actions. A sense of achievement, to be able to do something with an otherwise useless hand can be very motivating for patients with severe paresis. Furthermore, the multimodal feedback provided by the VR system may facilitate cerebral reorganization in a phase where highest plasticity can be expected [21].

In general, our patients were active during $72 \%$ their training sessions, which is more than identified in a previous review article where only $60 \%$ active time was found [10]. Although the exact dose-response of rehabilitative treatment approaches is still unclear, the results of several reviews indicate a beneficial effect of augmented training time on functional outcomes and activities of daily living [7-9]. It is recommended to find ways to increase therapy time after stroke and VR training seems to be an appropriate treatment alternative which also can increase adherence to exercises. Our intervention took place in a clinical setting under the supervision of a therapist, which facilitated adherence. However, in a home setting without supervision, it may be difficult to achieve recommend levels as demonstrated in a study by Standen et a. [22] Active time in therapy expressed as activity rate was the main focus of the current study. Measuring activity rate seems to be 
Table 2 Interaction of severity of paresis and Virtual Reality training

\begin{tabular}{|c|c|c|c|c|c|c|}
\hline \multirow{2}{*}{$\begin{array}{l}\text { Activity rate } \\
\text { Predictors }\end{array}$} & \multirow[b]{2}{*}{ B } & \multicolumn{2}{|c|}{ Univariate analysis } & \multirow[b]{2}{*}{$B$} & \multicolumn{2}{|c|}{ Multivariate analysis } \\
\hline & & $95 \% \mathrm{Cl}$ & $p$ & & $95 \%$ Cl & $p$ \\
\hline Severe paresis & -4.8 & $(-12.0,2.4)$ & .189 & -12.8 & $(-21.4,-4.1)$ & .005 \\
\hline VR & 10.3 & $(3.7,16.9)$ & .003 & 2.1 & $(-6.1,0.3)$ & .612 \\
\hline \multicolumn{7}{|l|}{ Interaction } \\
\hline (VR, severe paresis) & - & - & - & 15.1 & $(2.0,28.2)$ & .025 \\
\hline Active time & \multicolumn{3}{|c|}{ Univariate analysis } & \multicolumn{3}{|c|}{ Multivariate analysis } \\
\hline Predictors & B & $95 \% \mathrm{Cl}$ & $p$ & B & $95 \%$ Cl & $p$ \\
\hline Severe paresis & -106.5 & $(-456.6,243.7)$ & .544 & -595.7 & $(1022.9,-168.4)$ & .007 \\
\hline VR & 363.5 & $(30.8,696.1)$ & .033 & -107.0 & $(-511.5,297.4)$ & .597 \\
\hline \multicolumn{7}{|l|}{ Interaction } \\
\hline (VR, severe paresis) & - & - & - & 1057.1 & $(446.8,1667.4)$ & .001 \\
\hline Total time & \multicolumn{3}{|c|}{ Univariate analysis } & \multicolumn{3}{|c|}{ Multivariate analysis } \\
\hline Predictors & B & $95 \% \mathrm{Cl}$ & $p$ & B & $95 \% \mathrm{Cl}$ & $p$ \\
\hline Severe paresis & -34.9 & $(-353.2,283.4)$ & .826 & -418.5 & $(-840.2,3.2)$ & .052 \\
\hline VR & 141.0 & $(-172.5,454.6)$ & .370 & -214.7 & $(-613.9,184.4)$ & .285 \\
\hline \multicolumn{7}{|l|}{ Interaction } \\
\hline (VR, severe paresis) & - & - & - & 805.6 & $(203.3,1407.9)$ & .010 \\
\hline
\end{tabular}

the most objective and tangible aspect of motor training. Comparing VR to dose-matched interventions in terms of scheduled therapy sessions only may not reflect the real extent of activity. [23] There was no difference for total time and active time between VR and CT when looking at all patients, which was expected since the training in both groups was supposed to be matched for these parameters per protocol. However, for the more impaired patients also longer total time was revealed, not only a higher activity rate and longer active time. Longer sessions in VR may indicate that it was easier to perpetuate training for a longer time.

While our main focus was on training aspects related to time, we also intended to explore other features of the training provided in both groups. Counting repetitions for discrete functional movements was of very limited value in this study. The number of repetitions did not reflect activity rate or intensity, since both the $\mathrm{CT}$ and the VR training comprised continuous tasks, such as steering a plane (VR) or cleaning a table or writing (CT). VR comprised probably a larger part of continuous tasks that could not be counted as repetitions. Severely impaired patients with no grasp function frequently had no repetitions or no grasps registered, although they had been active through large parts of the training session. Training in VR consisted of exclusively functional tasks, while training in $\mathrm{CT}$ also comprised other tasks, mainly mobilization. However, repetitions of discrete functional movements registered were substantially, roughly 3 times, higher in both VR and CT than those registered by Kimberley et al. [24], who found only a mean 40.64 repetitions per session. An also relatively

Table 3 Content of tasks

\begin{tabular}{|c|c|c|}
\hline & VR & CT \\
\hline Content of tasks & Functional tasks only & $\begin{array}{l}\text { Functional tasks (65\%) strength exercises (10 \%) mobilization, } \\
\text { stretching ( } 23 \%) \text { other }(2 \%)\end{array}$ \\
\hline $\begin{array}{l}\text { Repetitions/session mean (SD), discrete } \\
\text { movements only }\end{array}$ & $130.05(217.11)$ & $128.87(85.10)$ \\
\hline \multirow[t]{2}{*}{ Unilateral/bilateral training per session } & 7.5 tasks unilateral (83 \%) & 4.9 tasks unilateral (73 \%) \\
\hline & 1.5 bilateral (17 \%) & 1.9 bilateral (27 \%) \\
\hline Use of real life objects & No & In $36 \%$ of the tasks \\
\hline \multirow[t]{2}{*}{ Feedback } & $\begin{array}{l}\text { Several modes of feedback, verbal, visual, } \\
\text { auditive, tactile }\end{array}$ & Verbal feedback only \\
\hline & $\begin{array}{l}\text { Knowledge of results and knowledge of } \\
\text { performance }\end{array}$ & Knowledge of results and knowledge of performance \\
\hline
\end{tabular}


low number of only 39 active and 12 purposeful movements per session were counted by Lang et al. [25]. Another study by Lang et al. revealed that functional UL movements occurred in $51 \%$ of training sessions [26]. Comparison with these earlier results indicates that our patients both achieved a very high activity rate and relatively many repetitions. Still, the activity rate and number of repetitions does not come close to what is regarded as facilitating neural plasticity based on animal studies [21, 27]. Activity rate seems to be the closest we can get to gauge intensity. However, it has been demonstrated that motor learning and cortical reorganization is not based on stereotype repetitions and the simple use of the hand [28]. Plastic changes only occur when skilled tasks are practiced, and may not automatically generalize to untrained tasks $[29,30]$. Motivation and engagement can facilitate general motor learning and retention [31]. It has been claimed that these requirements can be met by enriched virtual environments rather than by conventional therapy [2].

We also registered the kind of feedback delivered. The benefit of explicit feedback is still unclear. In some studies a beneficial effect of knowledge of performance on motor learning was found, while other studies suggest that explicit information interferes with implicit learning [32, 33]. In VR feedback was provided in a multi-modal way, since the patients could see scores reached, heard applause, felt vibrations on the hand. Also the training report that describes progress over a series of sessions can be regarded as feedback. Verbal feedback from the therapist comprising knowledge of performance and knowledge of results was provided in both groups. However, knowledge of performance with the intention to control for excessive compensatory trunk movement was provided in CT only. This may have allowed for more compensatory strategies in VR [32]. However, knowledge of performance can also be provided in VR by therapists or included as a part of the virtual environment as in the study by Subramanian et al. [34].

The fact that a session was video-recorded may have influenced both the patient and the therapist and could have resulted in altered behavior [35] and most likely in a way that effective training time was increased and small talk and extensive breaks discouraged. This may have affected CT more, since in VR the course of the sessions including breaks usually was pre-programmed. Since our study was cross-sectional we cannot tell if more was better in this case. Instead we have to wait for the results of the main study. The motor abilities of our patients were not assessed at the day of the videorecording. Therefore we cannot tell how much the initially severe motor impairment had improved that special day. We also have to acknowledge that the initial categorization based on active finger and wrist extension can be debated. However, we can tell from the videorecordings that patients with initially severe arm motor impairment still suffered from pronounced paresis at the day of recording. Although the video recordings were taken at a random day, we cannot rule out to have picked a session that was not quite representative for all sessions of this respective patient. Large deviations would have been reported by the therapist.

\section{Conclusion}

A markedly higher activity rate for patients with severe paresis in the VR group suggests that VR training facilitates more active training time. Also a longer active time and total time was registered for patients with severe impairments, indicating that it may be easier to adhere to the training. Only functional tasks were observed during training period in the VR group compared to only $65 \%$ of the time in the CT group. Several modes of feedback were registered in the VR group. It remains to find out if these differences in quality of training between the programs will cause any difference in arm function.

\section{Additional files}

Additional file 1: The scoring form. (PDF $145 \mathrm{~kb}$ )

Additional file 2: Data file. (XLS $25 \mathrm{~kb}$ )

\section{Abbreviations}

ARAT: Action Research Arm Test; CT: Conventonal training; FIM: Functional Independence Measure; VR: Virtual Reality training

\section{Acknowledgements}

We thank the participating patients and the research therapists at the participating sites, Gunhild Mo Hansen, Lola Quist Kristensen, John Wrigglesworth and Anette Lund. A special thanks to Kjersti Henriksen.

\section{Funding}

This research was supported by the Norwegian Research Council, award number 228792 and the Western Norwegian Health Authority, award number 911808 .

\section{Availability of data and materials}

Data are available as supplementary material (Additional file 2). Video recordings cannot be made available to preserve participant confidentiality.

\begin{abstract}
Authors' contributions
IB conceived of the study, participated in developing the design of the study, analysis and interpretation and drafted the manuscript. JS, HH, FB, LT, HP, GV participated in the design of the study, contributed to and critically appraised the manuscript and the revision. JA led the data analysis of the study. LT participated in data collection. All authors have read and acknowledged the final manuscript. All authors read and approved the final manuscript.
\end{abstract}

Competing interests

The authors declare that they have no competing interests.

Consent for publication

Not applicable. 


\section{Ethics approval and consent to participate}

The study was approved by the Regional Ethics Committee (ref. 2013/1663), REC South East, Oslo, Norway and all patients provided written informed consent.

\section{Author details}

'Department of Global Public Health and Primary Care, University of Bergen, Kalfarveien 31, 5018 Bergen, Norway. ${ }^{2}$ Department of Physical Medicine and Rehabilitation, Haukeland University Hospital, Bergen, Norway. ${ }^{3}$ Competence Center for Clinical Research, Haukeland University Hospital, Bergen 5012, Norway. ${ }^{4}$ Department of Research, Sunnaas Rehabilitation Hospital, Oslo, Norway. ${ }^{5}$ Department of Clinical Medicine, University of Oslo, Oslo, Norway. ${ }^{6} \mathrm{Hammel}$ Neurorehabilitation Centre and University Research Clinic, Voldbyvej 15, 8450 Hammel, Denmark. ${ }^{7}$ Rehabilitation Campus Sint-Ursula, Jessa Hospitals, Herk-de-Stad 3540, Belgium. ${ }^{8}$ Department of Rehabilitation Sciences, KU Leuven, Postbus 1501, Leuven 3000, Belgium.

Received: 31 May 2016 Accepted: 3 November 2016

Published online: 11 November 2016

\section{References}

1. Kleim JA, Jones TA. Principles of experience-dependent neural plasticity: implications for rehabilitation after brain damage. J Speech Lang Hear Res. 2008;51(1):S225-239.

2. Levin MF, Weiss PL, Keshner EA. Emergence of virtual reality as a tool for upper limb rehabilitation: incorporation of motor control and motor learning principles. Phys Ther. 2015;95(3):415-25.

3. Fluet GG, Deutsch JE. Virtual Reality for Sensorimotor Rehabilitation PostStroke: The Promise and Current State of the Field. Curr Phys Med Rehabil Rep. 2013;1(1):9-20.

4. Laver K, George S, Thomas S, Deutsch JE, Crotty M. Virtual reality for stroke rehabilitation: an abridged version of a Cochrane review. Eur J Phys Rehabil Med. 2015;51(4):497-506.

5. Saposnik G, Cohen LG, Mamdani M, Pooyania S, Ploughman M, Cheung D, Shaw J, Hall J, Nord P, Dukelow S, et al. Efficacy and safety of nonimmersive virtual reality exercising in stroke rehabilitation (EVREST): a randomised, multicentre, single-blind, controlled trial. Lancet Neurol. 2016; 15(10):1019-27

6. Veerbeek JM, Koolstra M, Ket JC, van Wegen EE, Kwakkel G. Effects of augmented exercise therapy on outcome of gait and gait-related activities in the first 6 months after stroke: a meta-analysis. Stroke. 2011;42(11):3311-5.

7. Kwakkel G, van Peppen R, Wagenaar RC, Wood Dauphinee S, Richards C, Ashburn A, Miller K, Lincoln N, Partridge C, Wellwood I, et al. Effects of augmented exercise therapy time after stroke: a meta-analysis. Stroke. 2004; 35(11):2529-39.

8. Lohse KR, Lang CE, Boyd LA. Is more better? Using metadata to explore dose response relationships in stroke rehabilitation. Stroke. 2014;45(7):2053-8.

9. Lang CE, Lohse KR, Birkenmeier RL. Dose and timing in neurorehabilitation: prescribing motor therapy after stroke. Curr Opin Neurol. 2015;28(6):549-55.

10. Kaur G, English C, Hillier S. How physically active are people with stroke in physiotherapy sessions aimed at improving motor function? A systematic review. Stroke Res Treat. 2012;2012:820673.

11. Kaur G, English C, Hillier S. Physiotherapists systematically overestimate the amount of time stroke survivors spend engaged in active therapy rehabilitation: an observational study. J Physiother. 2013:59(1):45-51.

12. Eng K, Siekierka E, Pyk P, Chevrier E, Hauser Y, Cameirao M, Holper L, Hagni K, Zimmerli L, Duff A, et al. Interactive visuo-motor therapy system for stroke rehabilitation. Med Biol Eng Comput. 2007;45(9):901-7.

13. Veerbeek JM, van Wegen $E$, van Peppen $R$, van der Wees PJ, Hendriks E, Rietberg M, Kwakkel G. What is the evidence for physical therapy poststroke? A systematic review and meta-analysis. PLoS One. 2014;9(2): e87987.

14. Timmermans AA, Spooren Al, Kingma H, Seelen HA. Influence of taskoriented training content on skilled arm-hand performance in stroke: a systematic review. Neurorehabil Neural Repair. 2010;24(9):858-70.

15. Rand D, Givon N, Weingarden H, Nota A, Zeilig G. Eliciting upper extremity purposeful movements using video games: a comparison with traditional therapy for stroke rehabilitation. Neurorehabil Neural Repair. 2014;28(8):733-9.

16. Yin CW, Sien NY, Ying LA, Chung SF, Tan May Leng D. Virtual reality for upper extremity rehabilitation in early stroke: a pilot randomized controlled trial. Clin Rehabil. 2014;28(11):1107-14.
17. Paquin K, Ali S, Carr K, Crawley J, McGowan C, Horton S. Effectiveness of commercial video gaming on fine motor control in chronic stroke within community-level rehabilitation. Disabil Rehabil. 2015;37(23):2184-91.

18. Levin MF, Snir O, Liebermann DG, Weingarden H, Weiss PL. Virtual reality versus conventional treatment of reaching ability in chronic stroke: clinical feasibility study. Neurol Ther. 2012;1(1):3.

19. Brunner I, Skouen JS, Hofstad H, Strand LI, Becker F, Sanders AM, Pallesen H, Kristensen T, Michielsen M, Verheyden G. Virtual reality training for upper extremity in subacute stroke (VIRTUES): study protocol for a randomized controlled multicenter trial. BMC Neurol. 2014;14:186

20. De Wit L, Kamsteegt H, Yadav B, Verheyden G, Feys H, De Weerdt W. Defining the content of individual physiotherapy and occupational therapy sessions for stroke patients in an inpatient rehabilitation setting Development, validation and inter-rater reliability of a scoring list. Clin Rehabil. 2007:21(5):450-9.

21. Murphy TH, Corbett D. Plasticity during stroke recovery: from synapse to behaviour. Nat Rev Neurosci. 2009:10(12):861-72.

22. Standen PJ, Threapleton K, Connell L, Richardson A, Brown DJ, Battersby S, Sutton CJ, Platts F. Patients' use of a home-based virtual reality system to provide rehabilitation of the upper limb following stroke. Phys Ther. 2015; 95(3):350-9.

23. Lohse KR, Hilderman CG, Cheung KL, Tatla S, Van der Loos HF. Virtual reality therapy for adults post-stroke: a systematic review and meta-analysis exploring virtual environments and commercial games in therapy. PLoS One. 2014;9(3):e93318.

24. Kimberley TJ, Samargia S, Moore LG, Shakya JK, Lang CE. Comparison of amounts and types of practice during rehabilitation for traumatic brain injury and stroke. J Rehabil Res Dev. 2010:47(9):851-62.

25. Lang CE, MacDonald JR, Gnip C. Counting repetitions: an observational study of outpatient therapy for people with hemiparesis post-stroke. J Neurol Phys Ther. 2007:31(1):3-10.

26. Lang CE, Macdonald JR, Reisman DS, Boyd L, Jacobson Kimberley $T$, Schindler-Ivens SM, Hornby TG, Ross SA, Scheets PL. Observation of amounts of movement practice provided during stroke rehabilitation. Arch Phys Med Rehabil. 2009:90(10):1692-8

27. Birkenmeier RL, Prager EM, Lang CE. Translating animal doses of taskspecific training to people with chronic stroke in 1-hour therapy sessions: a proof-of-concept study. Neurorehabil Neural Repair. 2010;24(7):620-35.

28. Nudo RJ. Recovery after brain injury: mechanisms and principles. Front Hum Neurosci. 2013;7:887.

29. Zeiler SR, Krakauer JW. The interaction between training and plasticity in the poststroke brain. Curr Opin Neurol. 2013;26(6):609-16.

30. Boyd LA, Vidoni ED, Wessel BD. Motor learning after stroke: is skill acquisition a prerequisite for contralesional neuroplastic change? Neurosci Lett. 2010;482(1):21-5.

31. Hodges NJ, Lohse KR, Wilson A, Lim SB, Mulligan D. Exploring the dynamic nature of contextual interference: previous experience affects current practice but not learning. J Mot Behav. 2014:46(6):455-67.

32. Cirstea CM, Ptito A, Levin MF. Feedback and cognition in arm motor skill reacquisition after stroke. Stroke. 2006:37(5):1237-42.

33. Boyd $L$, Winstein $C$. Explicit information interferes with implicit motor learning of both continuous and discrete movement tasks after stroke. Neurol Phys Ther. 2006;30(2):46-57. discussion 58-49.

34. Subramanian SK, Lourenco CB, Chilingaryan G, Sveistrup H, Levin MF. Arm motor recovery using a virtual reality intervention in chronic stroke: randomized control trial. Neurorehabil Neural Repair. 2013;27(1):13-23.

35. Spiers JA, Costantino M, Faucett J. Video technology. Use in nursing research. AAOHN J. 2000;48(3):119-24. 